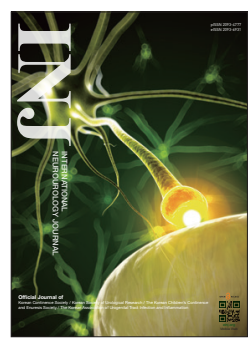

\title{
Reply to Commentary on "Changes in Prevalence and Treatment Pattern of Benign Prostatic Hyperplasia in Korea"
}

\author{
Jung Ki Jo1 ${ }^{1}$, Sung Ho Shinn², Kyu Shik Kim ${ }^{1}$, Hong Sang Moon ${ }^{1}$ \\ ${ }^{1}$ Department of Urology, Hanyang University College of Medicine, Seoul, Korea \\ ${ }^{2}$ Department of Thoracic and Cardiovascular Surgery, Cheju Halla General Hospital, Jeju, Korea
}

To the editor,

We would like to thank the authors of the detailed letter submitted responding to our article [1]. The incidence rate of benign prostatic hyperplasia (BPH) continues to increase as the elderly population increases, and treatment methods have continued to evolve accordingly [2]. Since BPH is one of the most common diseases affecting the quality of life of middle-aged and elderly men, urologists should be aware of it. When selecting the treatment method, it is necessary to take into account improvement in patient symptoms, side effects and complications.

Drug therapy is usually the first option for $\mathrm{BPH}$, but surgical treatment should be considered if the response to drug treatment is unsatisfactory or there are complications from $\mathrm{BPH}$ (urinary tract infection, bladder stone, hematuria, decreased kidney function, etc.). Although transurethral resection of prostate (TURP) has been widely used as the gold standard of surgical treatment, recent research has confirmed that holmium laser enucleation of the prostate (HoLEP) has become more common than TURP. Compared to TURP, HoLEP can remove almost all adenomas, so patients have the advantage of quicker improvement of symptoms and less bleeding after surgery [3,4]. On the other hand, HoLEP has a higher rate of postoperative incontinence than TURP, and has a steep operator learning curve [5]. In particular, inexperienced surgeons are likely to cause bladder injury during morcellation. Because of these disadvantages of HoLEP, it has not completely replaced TURP.

Many researchers have devised a number of methods for minimally invasive surgical treatments (MISTs) that are easier for surgeons to perform than TURP and HoLEP with a lower patient burden, some of which are still used for treatment. However, although a significant number of MISTs have reported good initial results, many are no longer in use. In some cases, the long-term follow-up results of MISTs showed high rates of recurrence and complications. MISTs are indispensable tools in the treatment of $\mathrm{BPH}$; however, these approaches must be proven through long-term follow-up in a number of studies with different operators.

We hope to perform further follow-up on treatment methods for $\mathrm{BPH}$ in South Korea in the future, to determine how the treatment pattern for $\mathrm{BPH}$ changes over time. Innovative treatment methods that are easy for urologists to learn, have fewer side effects and do not burden patients are needed.

- Conflict of Interest: No potential conflict of interest relevant to this article was reported.

\section{ORCID}

Jung Ki Jo

0000-0002-6080-7493

Sung Ho Shinn

0000-0001-9539-7941

Kyu Shik Kim

0000-0001-8755-0774

Hong Sang Moon

0000-0003-2101-1019

\section{REFERENCES}

1. Jo JK, Shinn SH, Kim KS, Moon HS. Changes in prevalence and treatment pattern of benign prostatic hyperplasia in Korea. Int Neurourol J 2021;25:347-54

2. Cai T, Wang N, Liang L, Zhou Z, Zhang Y, Cui Y. Meta-analysis of

Corresponding author: Hong Sang Moon (iD https://orcid.org/0000-0003-2101-1019 Department of Urology, Hanyang University Guri Hospital, 153 Gyeongchunro, Guri 11923, Korea

Email: moonuro@hanyang.ac.kr

Submitted: February 25, 2021 / Accepted after revision: March 7, 2021 
the efficacy and safety of imidafenacin for overactive bladder induced by benign prostatic hyperplasia in men receiving alphablocker therapy. Int Neurourol J 2020;24:365-74.

3. Oka S, Kobayashi K, Matsuda K, Takai K. Significance of membranous urethral length for recovery from postoperative urinary incontinence following holmium laser enucleation of the prostate. Int Neurourol J 2020;24:358-64.
4. Tae BS, Jeon BJ, Choi H, Park JY, Bae JH. A novel technique of morcellation using a pneumovesicum after holmium laser enucleation of the prostate in complicated situations: our initial experience and tips. Int Neurourol J 2019;23:46-55.

5. Cho JM, Moon KT, Yoo TK. Robotic simple prostatectomy: why and how? Int Neurourol J 2020;24:12-20. 